Predictors of Symptom Course in Alcohol Use Disorder

William E. Conlin ${ }^{1}$, Michaela Hoffman ${ }^{2}$, Douglas Steinley ${ }^{1}$, Alvaro Vergés ${ }^{3}$, \& Kenneth J. Sher ${ }^{1}$

${ }^{1}$ Department of Psychological Sciences, University of Missouri

${ }^{2}$ Department of Psychiatry and Behavioral Sciences, Medical University of South

Carolina

${ }^{3}$ Escuela de Psicología, Universidad Católica de Chile Pontificia

This research was supported in part by the NIH grants T32AA013526 and R01 AA024133 to Kenneth Sher. 


\begin{abstract}
Objective: Alcohol Use Disorder (AUD) has traditionally been viewed as a chronic, progressive, relapsing disorder (Jellinek, 1960; National Institute on Drug Abuse, 2018). However, little is known about the course of individual AUD criteria. To the extent that individual symptoms represent the focus of some treatments (e.g., withdrawal, craving), understanding the course of specific symptoms, and individual differences in symptom course, can inform treatment efforts and future research directions.
\end{abstract}

Method: The current study examined 34,653 participants form Wave 1 (2001-2002) and Wave 2 (2003-2004) of the National Epidemiological Survey on Alcohol and Related Conditions (NESARC; Grant, Moore, \& Kaplan, 2003; Grant, Kaplan, and Stinson, 2005), using logistic regression to analyze the extent to which AUD symptom course is predicted by heavy alcohol consumption, family history of alcoholism, and lifetime diagnosis of Conduct Disorder.

Results: The course of all AUD symptoms was significantly influenced by all four external criteria, with the magnitude of the prediction varying across different symptoms and different aspects of course.

Conclusion: The strength of the relationship appeared to be related to the theoretical proximity of a given predictor to AUD symptomatology, with heavy drinking being the strongest and family history of AUD being the weakest. The course of all AUD symptoms was strongly associated with the prevalence of the given symptom in the overall sample. Future work should include examining the interchangeability of AUD symptoms and considering heavy alcohol consumption as a criterion for AUD diagnosis.

Keywords: alcohol use disorder, alcohol use disorder course, symptom course, diagnosis 
Public Health Statement: This study found that the Family History of Alcohol Use Disorder, externalizing behavior, and excess drinking can influence the course of individual AUD symptoms. AUD symptoms are highly have very low chronicity in non-heavy drinkers. 


\section{Predictors of Alcohol Use Disorder Symptom Course}

Alcohol Use Disorder (AUD) and other substance-related addictions have traditionally been viewed as chronic, progressive, relapsing disorders (Jellinek, 1960; National Institute on Drug Abuse, 2018) and AUD is currently described by the National Institute on Alcohol Abuse and Alcoholism's website (e.g., "Alcohol Use Disorder," 2019, para. 1) as “a chronic relapsing brain disorder." In part, this viewpoint can be traced back to the Kraepelinian view of psychopathology, conceptualizing mental disorders as "disease entities" which remain relatively persistent over time (Kraepelin, 1907). In accordance with the disease model, research efforts have primarily focused on the course of AUD at the syndromal level (e.g., Grant et al., 2012; Lee et al., 2018; Vandiver \& Sher, 1991). Despite findings that individual symptoms vary over time, little attention has been given to how individual symptoms change and the factors associated with their course. Examining predictors of symptom course can provide information about the mechanisms underlying the temporal changes of individual AUD symptoms and may reveal that individual symptoms are more than just indicators of a latent syndrome.

\section{Syndrome Course vs Symptom Course}

The examination of syndrome course is an important tool for understanding the nature of psychiatric disorders. Much of what we know about risk, resilience, and progression of AUD has been learned by following the course of the syndrome over time (e.g., Bucholz et al., 2000; Grant et al., 2012; Timko et al., 2005; Rohde et al., 2001). However, much can be learned from the relatively unexplored course of symptoms. For example, imagine a mechanical watch that is running slow. We monitor the watch and observe that every 24 hours it is falling behind another 30 seconds. While this information is useful for assessing the condition of the watch, it would be 
premature to stop there. It would make sense to take apart the watch, examine the mainspring, the balance wheel, and other parts to more accurately understand which components are resulting in the phenomena of running "slow." However, rather than examining the constituent "parts" (symptoms), the past few decades of psychological science has primarily focused on syndromes (Kazdin, 1983; Lilienfeld \& Waldman, 2013). Recently, the burgeoning application of network analysis to symptoms (e.g., Borsboom \& Cramer, 2013) has renewed interest in the functional significance of individual symptoms as opposed to them primarily serving as indicators of an underlying latent variable. The symptom-focused approach offers new lens from which to view psychopathology and, with respect to addiction, might be a particularly valuable perspective given that it is consistent with neurobiological theories that postulate addiction etiology as a "cascade" of symptoms (see below).

\section{Theories of Addiction and the Role of Symptoms}

As noted by Bickel, Crabbe, and Sher (2019), in the Diagnostic and Statistical Manual of Mental Disorders, Fifth Edition (DSM-5, American Psychiatric Association, 2013), several symptoms appear to be tapping into clinical addiction concepts that correspond to key components of various prominent addiction theories, such as craving in incentive sensitization and withdrawal in allostasis. Examining the course of individual symptoms can provide support for theories based on the symptoms that appear to be core, stable features of the syndrome. It is useful to think of the symptom-syndrome-theory relationship in terms of Bleuler's (1911) description of primary and fundamental symptoms. Bleuler described primary symptoms as closer to the organic substrate of the disorder, whereas secondary symptoms are further downstream and occur as a result of the primary symptoms. Fundamental symptoms are those 
which are specifically characteristic of the disorder, whereas accessory symptoms are features that are shared with other disorders.

The allostasis perspective, one of the leading models of addiction, describes symptoms as having a "cascading" effect, following a cyclical process of preoccupation, binging, and withdrawal (Koob \& Le Moal, 2001). In Robinson and Berridge's (1993) incentive sensitization theory, drug cues become extremely salient, resulting in very high levels or "wanting" from the disordered individual. Leshner's (1997) "brain disease model," posits a metaphorical "switch" is thrown in the brain, resulting in a transition from voluntary drug use to involuntary and compulsive drug-taking behavior. In Piazza \& Deroche-Gamonet's (2013) multistep theory, addiction develops over the course of three distinct phases from recreational and sporadic, to intensified and sustained, and eventually into uncontrolled addiction. Each of these theories imply that certain key symptoms are both fundamental and primary and likely to drive the course of the other symptoms.

\section{Temporal Changes in Psychological Disorders}

The literature focused on the course of psychological disorders largely consists of studies examining changes at the syndromal level of disorders. As such, there is a strong body of evidence indicating that external variables can influence the syndromal course of AUD. The course of AUD has been found to vary significantly as a function of age, gender, and ethnicity (e.g., Grant et al., 2012; Timko et al., 2005), family history of alcoholism (Rohde et al., 2001) conduct problems (Bucholz et al., 2000), age of onset of drinking (Davis et al., 2019), and magnitude of alcohol consumption (e.g., Rohde et al., 2001; Schuckit \& Smith, 2011). Despite the extensive literature on the course of syndromal AUD, the literature examining the course of 
individual AUD symptoms is sparse. However, many studies have been conducted on the symptom course of various other forms of psychopathology, including PTSD (e.g., Pfaltz et al., 2010; Green et al., 1990), depression (e.g., Nivard et al., 2015; Conradi et al., 2011; Van Borkulo et al., 2015), and ADHD (e.g., Larsson et al., 2004; Hart et al., 1995), providing evidence that different symptoms have different longitudinal trajectories, with some of the variability in symptom course being accounted for by external predictors.

With evidence of genetic and environmental influences of individual symptom course in other forms of psychopathology, one can reasonably speculate that AUD symptom course is also influenced by exogenous predictors. However, relatively few studies have examined this hypothesis. O’Neill \& Sher (2000) examined stability of tolerance and withdrawal and found that the persistence of both symptoms decreased considerably over time. Additionally, the prevalence of these symptoms decreased even among individuals with stable heavy consumption patterns. A recent study by Vergés and colleagues (2020) examined symptom course from a developmental perspective. This study found the symptoms indicating attempts at quitting/controlling drinking and continuing to drink despite physical/psychological problems were less persistent and less predictive of AUD course in younger adults, possibly suggesting greater rates of false positive endorsement among younger adults. Although the study aimed to evaluate the possibility that the age-prevalence curve of AUD could be in part due to false positive diagnoses being highly prevalent in early adulthood, it revealed important information about the variability of symptom course across individual symptoms and across time.

Due to the dichotomous nature of symptoms in widely used diagnostic systems, there are a limited number of ways to define symptom course over two measurement occasions. On a 
follow-up measurement occasion, a symptom can be found to be present among those who exhibited the same symptom at the prior measurement occasion (persistence), can be present among those who did not exhibit the symptom and report never having experienced the symptom at the prior measurement occasion (onset), or can be exhibited among those who did not exhibit the symptom at the prior measurement occasion but did report a history of the symptom prior to that assessment (recurrence). While these elements of "course" have received a variety of different names (e.g., stability, chronicity, incidence, relapse, etc.), the majority of the studies in the psychopathology literature examine course through one or more of these three general perspectives. When studying course, it should be noted that the nature of how "course" is defined has implications for the sample, with symptomatic individuals in persistence samples, presymptomatic individuals in onset samples, and previously symptomatic individuals in recurrence samples.

\section{Predictors of Symptom Course}

The current study examined changes in symptom course associated with having a family history of alcoholism, externalizing behavior, binge drinking, and heavy drinking. To limit the already large number of tests, we selected the four predictors which we hypothesized would have the most robust effects and theoretical relevance. To our knowledge, this is the first study to examine how these predictors influence the course of individual AUD symptoms. The selected predictors have been established as modifiers of syndromal AUD course (e.g., Bucholz et al., 2000; Rohde et al., 2001; Schuckit \& Smith, 2011). As such, these predictors were expected to have effects on the course of individual AUD symptoms. Further, these predictors were chosen to "map" different symptoms that would be theoretically related to the given predictor. Research 
has identified that some AUD symptoms (e.g., Social/Interpersonal, Failure to Fulfill) are more related to externalizing behavior than others (McDowell et al., 2019), some symptoms (e.g., Craving, Larger/Longer) are more closely associated with binge drinking (Morgenstern et al., 2016), and other symptoms (Tolerance and Withdrawal) are posited to be neuroadaptational changes resulting from prolonged exposure to alcohol (e.g., Koob, 2011). To contrast the differential between-symptom effects expected from the other predictors, a proxy genetic predictor (family history of AUD) was expected to have less differential between-symptom effects than other predictors.

Understanding symptom course in different at-risk populations of drinkers can have important implications for how we view AUD. Examining these predictors of course may help identify symptoms that are particularly stable within different subgroups of drinkers (e.g., externalizing drinkers vs. non-externalizing drinkers). This may also help elucidate how different presentations arise out of the core features of AUD. Such symptoms have been suggested in the literature for a variety of disorders including depression (e.g., Nutt et al., 2008), PTSD (e.g., Braun et al., 1990) and bulimia nervosa (Levinson et al., 2017), and AUD (Langenbucher et al., 2000). Given the heterogeneity of presentations that could meet criteria for AUD (e.g., Lane \& Sher, 2015), understanding predictors of symptom course in AUD may help identify different primary/fundamental symptoms and the mechanism by which secondary/accessory symptoms crystalize in different subgroups of drinkers. By identifying and understanding these mechanisms, it may be possible to focus treatment on the specific symptom and disrupt the entire process, ultimately ameliorating many symptoms of the disorder. Based on contemporary theories of addiction emphasizing neurobiological adaptions related to prolonged alcohol exposure (e.g., Koob, 2011) and compulsive use (e.g., Lüscher et al., 2020) as core features of 
that define addition, we postulate that the symptoms most fundamental to AUD are Withdrawal and Quit/Cut Down. We expect these fundamental symptoms to onset at lower rates (due to less overlap with other forms of psychopathology) but have higher rates of persistence. Some literature suggests that AUD symptoms are associated with different degrees of severity (e.g., Boness et al. 2019, Lane \& Sher, 2015) Based on these studies, we expect more "severe" symptoms (e.g., Give Up, Withdrawal, and Failure to Fulfill to have high persistence and relatively high recurrence rate compared to their onset rate. Once the disorder has manifested in a severe form, we would expect greater chronicity, and upon remission, we would expected these more disordered individuals to relapse into severe symptoms at a higher rate than the rate in which they were acquired. While we expect recurrence rates to be greater than onset rates for all symptoms, we hypothesize that the rates will be relatively higher for the more severe symptoms.

We expected that the predictors will have differential effects on the symptoms based on their nomological relevance to the given symptom. We expected Conduct Disorder to more strongly influence the course of “externalizing” AUD symptoms (i.e., Hazardous Use, Social/Interpersonal, and Failure to Fulfill). Binge drinking was expected to have stronger effects on Hazardous Use and Larger/Longer, while heavy drinking was expected to have strong effects on Tolerance, Withdrawal, and Physical/Psychological Problems. As noted, Family History was expected to have similar effects across all symptoms.

\section{Method}

Data from the National Epidemiological Study on Alcohol and Related Conditions (NESARC) study was analyzed to examine the effects that family history of alcoholism, 
externalizing behavior, and quantity/frequency of alcohol consumption have on the course of individual AUD symptoms. NESARC is a nationally representative sample of 43,093 participants, 18 years and older, conducted by National Institute on Alcohol Abuse and Alcoholism (NIAAA) in 2001-2002 (Grant et al., 2003) and again in 2004-2005 (Grant et al., 2005). Wave 2 was an attempted follow up at all of the 43,093 participants who participated in Wave 1 of NESARC. Response rate was $86.7 \%$, resulting in a final count of 34,653 participants. Diagnosis and symptoms were measured by the NIAAA Alcohol Use Disorder and Associated Disabilities Interview Schedule- DSM-IV Version (AUDADIS-IV), which has extensive evidence supporting its reliability and validity. (e.g., Grant et al., 2001; Grant et al., 2003). Participant characteristics and survey methodology for NESARC has been described in detail elsewhere (Grant et al., 2004).

\section{Predictors}

For the purpose of this study, Family History was defined as having at least one parent or adult living in the home with an alcohol use disorder (alcohol abuse or alcohol dependence, per the AUDADIS-IV). We operationalized externalizing behavior as the presence of a lifetime diagnosis of Conduct Disorder. Conduct Disorder provides a behavioral operationalization of antisocial traits and captures a fixed period of life that all participants have already passed through. Heavy drinking corresponds to the NIAAA (2019) guidelines for risky alcohol consumption. These guidelines define risky drinking as " 4 or more drinks on any day or 8 or more drinks per week for women and 5 or more drinks on any day or 15 or more drinks per week for men". We utilized two alcohol consumption variables, one for binge drinking, or Exceeding Daily Limits (EDL), and one for Exceeding Weekly Limits (EWL). These consumption variables 
has considerable overlap, with $75 \%$ of individuals meeting for EWL also meeting for EDL and $33 \%$ of individuals meeting for EDL also meeting for EWL, resulting in a total of 30\% overlap. Consumption measures were analyzed separately as two different high-risk alcohol consumption patterns.

\section{Symptom Course}

For analytic purposes, we only used participants who provided data at both waves of NESARC $(N=34,653)$. Table 1 shows the method by which we defined persistence, onset, and recurrence. For persistence analysis, we used participants who endorsed a given past-12-month symptom at Wave 1. For onset analyses, we analyzed participants who did not have a lifetime endorsement of the symptoms (i.e., negative for past 12 months and for prior to past year). For symptom recurrence, we analyzed participants who had the symptom prior to the past year at Wave 1 but did not report the symptom during the past 12 months at Wave 1 . For all three analyses, the participants were only included if they met the stated condition and reported drinking in the past year prior to Wave 2. Endorsing a symptom at a given wave is defined by experiencing the symptom within the past 12 months, as is standard for diagnosis in commonly used diagnostic manuals (e.g., DSM-IV, DSM-5) (American Psychiatric Association, 1994, 2013). The symptoms included in our analyses are 10 of the 11 DSM-5 AUD symptoms ${ }^{1}$, with the omission being Craving, as Craving was not assessed at Wave 1 of NESARC.

\section{Analytic Approach}

To account for the complex sampling design used in NESARC, the SAS procedure SURVEYLOGISTIC was used to determine odds ratios for symptom persistence, onset, and recurrence. This procedure accounted for the primary sampling unit, stratum, and sampling 
weights of the NESARC data using Wave 2 sampling weights. To assess the predictors of course, data were analyzed separately for each predictor, with the exception of age and sex, which were included in all analyses. Separate sets of analyses were conducted for each "stage" of course (i.e., persistence, onset, and recurrence), along with each symptom and predictor, allowing us to examine the effects of each predictor on the course of each symptom. False discovery rate was used to control for familywise error rate. After completing these analyses on symptom course, additional analyses were conducted with Family History, Conduct Disorder, EWL, sex, and age included together in each model to test for unique prediction. EDL was excluded from these analyses due to collinearity between EDL and EWL.

\section{Results}

Due to the large number of models estimated, we will not discuss every finding in detail. Rather, we discuss the general trends found in the data and include a table in the Supplementary Materials which comprehensively details all analyses. Using false discovery rate to correct for multiple statistical tests, significant results $(\mathrm{p}<.05)$ were found for $89 \%(107 / 120)$ of the total number of logistic regression analyses conducted. For symptom persistence (i.e., endorsed the symptom at Wave 1 and Wave 2), significant results were found in 85\% (34/40) of the models. For symptom onset (i.e., never had the symptom at Wave 1, then endorsed the symptom at Wave 2), significant results were found for $100 \%$ (40/40) of the models. For symptom recurrence (i.e., had the symptom prior to Wave 1, did not endorse the symptom at Wave 1, then endorsed the symptom at Wave 2), significant results were found for 83\% (33/40) of the models. Across all analyses (persistence, onset, and recurrence), significant results ( $\mathrm{p}<.05)$ were found for Conduct Disorder, Family History, EDL, and EWL, in 76\% (23/30), 87\% (26/30), 93\% (28/30), and $100 \%(30 / 30)$ models, respectively. 
Persistence. Symptoms were highly unstable between Wave 1 and Wave 2, with persistence ranging from $20.28 \%$ for Failure to Fulfill to $42.17 \%$ for Hazardous Use, closely followed by Larger/Longer (42.02\%, SE=0.66\%) and Quit/Cut Down (41.15\%, SE=0.57\%). The strongest predictor of Hazardous Use persistence was EDL, with 48.02\% (SE=0.81\%) of EDL drinkers reendorsing Hazardous Use at Wave 2 compared to $26.65 \%$ (SE=0.87\%) of non-EDL drinkers $(\mathrm{OR}=2.34, \mathrm{P}<0.01)$. The least persistent symptom, Failure to Fulfill, was best predicted by EWL, with $26.72 \%$ (SE=1.24\%) of EWL drinkers reendorsing the symptom compared to only $4.7 \%(\mathrm{SE}=0.53 \%)$ reendorsement in non-EWL drinkers $(\mathrm{OR}=7.25, \mathrm{P}<0.01)$.

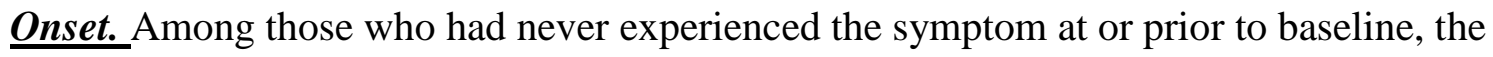
onset of new symptoms between Wave 1 and Wave 2, varied from $0.63 \%(\mathrm{SE}=0.02 \%)$ in Give Up to $6.95 \%(\mathrm{SE}=0.10 \%)$ in Larger/Longer. The four predictors had the strongest effects on symptom onset (median $\mathrm{OR}=2.47$ ), compared to persistence (median $\mathrm{OR}=1.63$ ) and recurrence (median $\mathrm{OR}=1.92$ ). Heavy drinking (EWL and EDL) emerged as the most robust predictor of onset, with EWL being the strongest predictor for 8 symptoms and EDL being the strongest predictor for the remaining 2 symptoms. The strongest predictor of the onset of Larger/Longer was EWL, with a $20.63 \%$ ( $\mathrm{SE}=0.53 \%$ ) onset rate in EWL drinkers compared to the $8.58 \%$ $(\mathrm{SE}=0.14 \%)$ onset rate in non-EWL drinkers $(\mathrm{OR}=3.16, \mathrm{P}<0.01)$. The strongest predictor for onset of Give Up was EDL, with EDL drinkers having a hazard rate of $1.63 \%$ (SE=0.09\%) compared to only $0.28 \%$ ( $\mathrm{SE}=0.03 \%, \mathrm{OR}=4.45, \mathrm{P}<0.01)$ in non-EDL drinkers.

Recurrence. Symptom recurrence ranged from $18.34 \%$ ( $\mathrm{SE}=0.38 \%)$ for Larger/Longer to $5.73 \%(\mathrm{SE}=.43 \%)$ for Failure to Fulfill and $5.73 \%(\mathrm{SE}=0.29 \%)$ for Time Spent. The strongest predictor of Larger/Longer was EDL, with binge drinkers experiencing a recurrence rate of 
$27.92 \%(\mathrm{SE}=0.52 \%)$ and non-binge drinkers experiencing a recurrence rate of $10.45 \%$

( $\mathrm{SE}=0.45 \%, \mathrm{OR}=2.73, \mathrm{P}<0.01$ ). EDL was also the strongest predictor for Failure to Fulfill, with $7.73 \%$ ( $\mathrm{SE}=0.53 \%$ ) of binge drinkers having Failure to Fulfill recurrence compared to $2.39 \%$ ( $\mathrm{SE}=0.04 \%)$ of non-binge drinkers $(\mathrm{OR}=3.23, \mathrm{P}<0.01)$. All four predictors were nearly equal in predicting the recurrence of Time Spent, with odds ratios between 2.49 and 2.59.

\section{Magnitude of effects}

Figure 1, Figure 2, and Figure 3 display the odds ratios for each set of analyses. The strongest overall effect across analyses was EWL (OR range $=1.34-7.25$, median OR $=2.65)$. EDL $(\mathrm{OR}$ range $=0.75-4.45$, median $\mathrm{OR}=2.35)$ had comparable effects, while Conduct Disorder $(\mathrm{OR}$ range $=1.01-3.51$, median $\mathrm{OR}=1.63)$ and Family History $(\mathrm{OR}$ range $=1.05-5.58$, median $\mathrm{OR}=1.48$ ) had relatively smaller effects. While the alcohol consumption variables (EDL, EWL) were the strongest overall predictors, their effects were most pronounced in the onset of new symptoms. In the persistence analyses, we see relatively equal effects between EWL (median OR $=1.81$ ), $\mathrm{EDL}$ (median $\mathrm{OR}=1.44$ ), Conduct Disorder (median $\mathrm{OR}=1.61$ ) and Family History (median $\mathrm{OR}=1.58$ ). In the onset analyses, the median ORs for EWL and EDL were 3.53 and 3.15, respectively, while Conduct Disorder (median OR=1.63) and Family History (median $\mathrm{OR}=1.48$ ) had considerably weaker effects. In the recurrence of symptoms, consumption variables were stronger predictors ( $\mathrm{EWL}$ median $\mathrm{OR}=2.64$, $\mathrm{EDL}$ median $\mathrm{OR}=2.66$, Conduct Disorder median $\mathrm{OR}=1.43$, Family History median $\mathrm{OR}=1.43$ ), but with a less pronounced difference than in the onset of new symptoms.

The multiple predictor analyses found that almost all comparisons accounted for unique variance. All single predictor models that were significant in the onset and recurrence analyses 
were also significant as multiple predictor models. For persistence, the only effects that were no longer significant in the multiple predictor models were Family History on Tolerance, EWL on Time Spent, and Family History on Give Up. These changes did not appear to have any theoretical implications, but rather appeared due to the attenuation of already relatively small effects found in the single predictor models.

\section{Symptom Prevalence and Symptom Course: The Influence of Base Rates}

Ancillary analyses found that the prevalence of a given symptom at baseline (Wave 1) was significantly associated with the persistence $\left(r=.90, r_{s}=.87\right)$, onset $\left(r=.99, r_{s}=1.00\right)$, and recurrence $\left(r=.91, r_{s}=.89\right)$ of the symptom (see Supplemental Figure 1, i.e. the prevalence at Wave 2). Additional analyses were conducted on strength of this association when conditioned on the four external predictors (see Supplemental Figures 1-4). These analyses revealed that, although symptoms are affected differently by the four predictors, the overall pattern of symptom course is inextricably tied to the base rate of a given symptom. Tolerance was found to be consistently less stable than would be expected by the prevalence, while Physical/Psychological Problems was found to be more stable than would be expected.

Although not an initial goal of the current study, we observed very low symptom stability in non-heavy drinkers. Along with the results found in the supplementary table, additional analyses were conducted to view the stability of the symptoms in individuals who were neither EDL nor EWL. In these moderate drinking individuals, symptom stability was even lower, with stability ranging from $34.4 \%$ ( $\mathrm{SE}=.44$ ) for Quit/Cut Down, to $2.7 \%$ (SE=N/A) for Failure to Fulfill. The standard error was unable to be estimated in Failure to Fulfill due to the very few number of individuals in whom the symptom persisted. 


\section{Discussion}

Given the recent interest in symptoms as meaningful entities in their own right and not just indicators of a latent variable, we aimed to examine the symptom-level course of AUD and explore potential predictors that may alter the trajectory of individual symptoms. The results indicate that factors generally associated with the course of syndromal AUD are also associated with each individual symptom but vary considerably by symptom and type of course (onset, persistence, or recurrence). We hypothesized that predictors which are nomologically related to specific symptoms would have stronger effects on these symptoms (e.g., externalizing behavior would more strongly affect the course of Social/Interpersonal problems), but the results provided only modest support for this hypothesis. However, the results of this study do indicate that external criteria do significantly affect the course of AUD symptoms relative to their conceptual and temporal proximity to behavioral manifestations of AUD. Family History, the most conceptually distal predictor, had the smallest effects, followed by Conduct Disorder with medium effects and heavy alcohol consumption with the strongest effects on symptom course. ). The findings of the current study provide evidence that the course of individual symptoms may be especially associated with external factors that shape the manifestation of AUD in a given individual. However, many of the overall patterns remained similar across different symptoms, despite the variability in the symptoms, indicating that there may be an underlying common cause undergirding course. These results suggest that an optimal approach may be method which takes into consideration the significance of individual symptoms, but does not wholly abandon the concept of a latent entity. The findings also have important implications regarding AUD prognosis, as they suggest that those most at-risk for the development of AUD also have similar patterns of risk for chronic and recurring symptoms. 


\section{Symptom Onset}

Results for symptom onset showed the most discernable trends, as well as the greatest support for our hypothesis. Alcohol consumption (EDL, EWL) was the strongest predictor for the onset of all ten symptoms, and EDL/EWL were the two strongest predictors in nine of the ten symptoms. While most symptoms had EDL and EWL creating similar risk of onset, EWL was considerably stronger in Physical/Psychological Problems (EDL OR=3.12, EWL OR=4.27) Tolerance (EDL OR=1.60, EWL OR=2.26), and Quit/Cut Down (EDL OR=2.41, EWL $\mathrm{OR}=3.36)$. The especially elevated risk for Physical/Psychological Problems and Tolerance was consistent with our hypotheses about symptoms related to prolonged heavy drinking. Also as expected, EWL was also the strongest predictor of Withdrawal, however, this effect was not as different from EDL (EDL OR=2.33, EWL OR=2.63) as in the aforementioned symptoms.

The results for Conduct Disorder partially aligned with our hypothesis. As expected, Conduct Disorder had especially strong prediction on the onset of Social/Interpersonal Problems and Failure to Fulfill. Prediction of Hazardous Use was low, but this is consistent with other findings regarding the relatedness of Hazardous Use and externalizing (McDowell et al., 2019). Unexpectedly, Conduct Disorder also strongly predicted the onset of Give Up. This may be explained by the higher severity threshold for Give Up. Giving up important activities to drink is indicative of compulsive use, which is thought to be the most severe stage of addiction (Piazza \& Deroche-Gamonet, 2013). Previous studies have found that Give Up is associated with higher severity of AUD and Give Up has been identified as the most severe (as indicated by IRT difficulty parameter) symptom of AUD (Boness et al., 2019; Lane et al., 2016). The current 
study also found a positive association between Conduct Disorder and AUD severity, which may explain the association between Conduct Disorder and onset of Give Up.

\section{Symptom Persistence}

The current study found that AUD symptoms are highly unstable over time, indicating that more attention should be given to the course of individual symptoms in future research on AUD. Conduct Disorder did not predict persistence of "externalizing symptoms" as expected and did not mirror the effects seen in symptom onset. Conduct Disorder did not significantly predict the persistence of Failure to Fulfill, and the magnitude of prediction in Social/Interpersonal Problems, while significant, was roughly equivalent to the effects present in other less “externalizing" symptoms. One possible explanation for this finding is the relatively small number of individuals present in certain analyses due to the design of the study. This problem is discussed in greater detail in the limitations section.

EDL, which is a strong predictor of onset and recurrence, was a relatively weak predictor of persistence. This may suggest that people who binge drink experience adverse consequences from their alcohol use, but these symptoms are relatively fleeting consequences of overindulgence rather than the result of the neuroadaptational changes characteristic of AUD. Consistent with this idea, EDL is the strongest predictor of persistence of Hazardous Use and Larger/Longer, while failing to significantly predict the persistence of Tolerance and Physical/Psychological Problems. Unexpectedly, EDL drinkers were significantly less likely to report Time Spent persistence than non-EDL drinkers. This may be also explained by the previously suggested account, as non-alcoholic binge drinkers may have noticed that their alcohol binges were becoming excessively time consuming and corrected the behavior. Follow- 
up analyses supported this interpretation, as EDL drinkers who endorsed Time Spent at Wave 1 had considerably decreased their alcohol consumption at Wave 2, whereas non-EDL drinkers had increased their alcohol consumption. This may have clinical implications, as individuals with problematic drinking behavior may be more responsive to interventions which highlight the excessive amount of time they spend consuming and recovering from the effects of drinking (similar to smoking interventions that highlight the amount of money saved by not smoking).

EWL significantly increased the persistence of all AUD symptoms, which supports theories of addiction that conceptualize AUD as a neuroadaptational change that occurs with consistent exposure to high quantities of alcohol. The findings regarding EDL and EWL suggest that alcohol consumption patterns may be related to the persistence of some symptoms more so than others. As displayed in Figure 1, EDL seems to have unique effects on certain symptoms, whereas EWL presents a more consistent general risk for persistence across all symptoms.

\section{Symptom Recurrence}

Due to the pitfalls of lifetime recollection (e.g., Haeny, et al., 2016; Moffitt et al., 2010) and the relatively low number of individuals in some of the recurrence analyses, we will avoid making overly granular interpretations about the effects of individual predictors on the recurrence of individual symptoms. Generally, we found that the four external predictors divided into two groups. The consumption variables tended to have similar magnitudes of prediction, while Family History and Conduct Disorder tended to have a similar, albeit lower, magnitudes of prediction. This is consistent with previous studies on AUD (e.g., Tuithof et al., 2014) which found that heavy alcohol consumption is linked to AUD recurrence in individuals who previously had an AUD. One notable exception is Time Spent, which had nearly equivalent 
recurrence rates for all four predictors. Among those who experienced a recurrence of Time Spent, $100 \%$ of the individuals also met diagnostic criteria for having AUD recurrence. This may suggest that Time Spent is a fundamental symptom in the process of AUD relapse, at least among those who had previously experienced the symptom.

\section{Influence of Prevalence}

The ancillary analyses were conducted with the intention of examining how the each external predictor differentially affected each individual symptom. However, regardless of the unique effect of each predictor on the symptom, the prevalence of any given symptom is the strongest predictor of the course of that symptom. Although it makes mathematical sense that higher frequency events are more likely to be observed at any given timepoint, the magnitude of this effect was striking. Despite significant changes in symptom course due to the predictors, the symptoms' course tended to cluster into high (Quit/Cut Down, Larger/Longer, Hazardous Use), medium (Withdrawal, Physical/Psychological Problems) and low (Social/Interpersonal Problems, Time Spent, Give Up, and Failure to Fulfill) groups across all analyses, largely as a function of prevalence. As such, it appears that the "stickiness" of a given symptom cannot be determined independently of the symptom's prevalence.

Against the background of base rate determinism, these analyses yielded some noteworthy findings. Tolerance was a particularly inconsistent symptom, and has been previously suggested to have suboptimal validity (e.g., Chung \& Martin, 2005) as assessed in diagnostic interview. Consistent with our overall findings, binge drinking appears to have a unique relationship with the persistence of Hazardous Use and Social/Interpersonal Problems. In individuals who binge drink, Hazardous Use and Social/Interpersonal problems are more 
persistent than would be expected given their prevalence, whereas in non-binge drinkers those symptoms are less persistent than would be expected given their prevalence. Also consistent with overall findings, heavy consumption appears to be the strongest determinant of symptom recurrence for all symptoms. For some symptoms, recurrence in non-heavy drinkers is equal to less than the prevalence of the symptom (Supplemental Figure 4).

\section{Limitations}

As noted, self-report data for lifetime recollection tends to have questionable accuracy, especially in regards to AUD symptoms (Haeny, et al., 2016). A recent study also indicated that self-reported family history of AUD may have also poor sensitivity (Andreasen et al., 1986; Schuckit, et al., 2020). There may have been more Family History than reported, and the true effects of Family History may be stronger than those found by our study. Another limitation was the usage of only 10 of the 11 AUD criteria. As the first wave of NESARC only assessed DSMIV criteria, we were unable to analyze the DSM-5 diagnostic criterion "craving." The now obsolete criterion "legal consequences due to drinking" was dropped from the analyses, for reasons described by Hasin and colleagues (2013). Noted in detail below, future research should examine the course of craving.

It should be noted that the large number of participants resulted in some statistically significant effects that may not offer any clinically meaningful interpretation. For example, Conduct Disorder significantly predicts the onset of the Quit/Cut Down. However, the size of the effect is marginal, with the lower bound of the confidence interval of the odds ratio being 1.05. We caution against over-interpreting such effects and encourage readers to view the full table provided in the Supplementary Material. Inversely, there are also some analyses that relied on individuals simultaneously endorsing two different low base rate phenomena (e.g., Failure to 
Fulfill and Conduct Disorder). While the NESARC sample is large enough to produce estimates for such effects, they were estimated from subpopulations containing as few as 33 people. Future replications will be needed to ensure that the effects discovered in these sparse subpopulations are robust enough for valid interpretation.

\section{Implications}

Our findings regarding lack of symptom stability highlight the importance of monitoring individual symptoms of AUD. As different symptoms occur and desist frequently, questions arise about the interchangeability of symptoms in syndromal AUD. For example, imagine an individual initially met criteria for AUD by endorsing Tolerance, Larger/Longer, and Hazardous Use. In a follow-up assessment, this individual endorsed Withdrawal, Quit/Cut Down, and Failure to Fulfill. From a purely diagnostic standpoint, these would be equivalent diagnoses, whereas a symptom-informed approach may suggest a progression of AUD over time. While the evidence of this study is not sufficient to propose a hierarchical model of AUD symptoms, varying stability (or lack thereof) found in AUD symptoms raises concerns about simple symptom count approaches to diagnosis (see also Lane \& Sher, 2015). In contrast, the relatively consistent overall patterns between symptoms and between persistence, onset, and recurrence indicate that a mixed approach incorporating both an underlying common cause and individual symptom effects may be provide a compelling avenue for future research.

Given our findings on the lack of symptom stability in non-heavy drinkers, we believe it may be important to consider including a minimum consumption requirement in future revisions of the diagnostic criteria. The notion that measures of heavy consumption can supplement and improve the validity of diagnosis has been previously suggested in the AUD literature (e.g., 
Hoeppner et al., 2011; Saha et al., 2007). In our findings, these suggestions are supported by the very low symptom stability in individuals who did not exceed daily or weekly limits. These findings suggest that many individuals w ho endorse a symptom in the absence of a heavy drinking pattern may be providing false positive responses. If these responses do actually represent true positives, the lack of temporal stability would indicate that these "symptoms" in moderate alcohol consumers do not represent a chronic relapsing disease, but rather a transient consequence that may more closely be related to circumstances than to an underlying psychopathology. As noted by Martin and colleagues (2014), treating consequences as a symptom of AUD reduces the validity of the diagnosis. That being said, we acknowledge that certain populations may experience serious consequences from relatively low amounts of alcohol consumption (e.g., adolescents, pregnant women, etc.), and the incorporation of a "consumption cutoff" would require further research.

Another important finding for the future of AUD research is the strength of the effect that the base rate has on the chronicity of a given symptom. As previously noted, the vast majority of variability in a given symptom's course can be accounted for by the overall base rate of the symptom. Regardless of external criterion, the rank-order relationship between symptom course and base rate was almost completely unchanged. Future research on AUD symptom course should not only make note of this relationship, but actively seek to disentangle the true "stickiness" of a symptom from simple base rate effects. Until such effects can be isolated, the most prevalent (and presumably least severe) symptoms will continually rise the forefront symptom-focused analysis simply on the basis of prevalence, obfuscating which symptoms are truly the most fundamental to a given disorder. Future work on clinical samples may help us 
"beat the base rate" and identify symptoms that are inherently more persistent than others, independent of their prevalence.

From a clinical standpoint, significant gains could be made from bettering our understanding of symptom course. If certain symptoms are more persistent in certain types of drinkers, it may be useful to develop new types of interventions that can help members of that subpopulation to overcome that particular symptom. For example, the current study suggests that a binge drinker may benefit from treatment focused on moderation and reducing hazardous activities while drinking, whereas a chronic daily drinker may need to address physical/psychological problems exacerbated by drinking.

With the increasing use of precision medicine and symptom-specific interventions, it is important that researchers understand the factors that influence the retention and remission of different symptoms (Heilig \& Egli, 2006; Volpicelli et al., 1992). Given the usage of naltrexone to reduce cravings when treating alcohol or drug dependence (e.g., Hendershot et al., 2017; Volpicelli et al., 1992), future research on symptom course should examine the course of craving. If craving is an unstable symptom, particularly in certain subgroups of drinkers, it may not be the ideal symptom to focus on when treating AUD. Conversely, if certain types of drinkers demonstrate markedly greater risk to experience a recurrence in craving, these drinkers may be the most suitable candidates for naltrexone-assisted treatment. The current research indicating that most AUD symptoms desist after a period of time suggests that resources and effort should be spent targeting symptoms that are most persistent.

\section{Conclusion}


Ultimately, the current study provides additional evidence for the remarkable heterogeneity of AUD in its current diagnostic form. The symptoms that constitute AUD vary considerably in their prevalence, course, and the extent to which external forces influence them over time. In part, the strength of this influence appears to relate to how proximal a given risk factor is to the behavioral outcome of AUD. However, in many instances, the external predictors that are conceptually related to certain symptoms appear to have significant influence on the onset, persistence, and recurrence of these symptom, leading to differential prediction of symptoms that goes beyond the overall pattern associated with the proximity of predictors. The significance of heavy alcohol consumption in the development and maintenance of AUD symptoms provides further rationale for the incorporation of consumption measures alongside or within the diagnostic criteria for AUD. However, regardless of the robustness of external predictors of symptom course, the overall prevalence of a given symptom will often be the strongest determinant of symptom course, at least in general population samples. As the field continues to uncover more about the "lives" of individual symptoms, and clinical intervention moves closer towards individualized medicine, additional research will be necessary to understand how individual symptoms interact and manifest into the set of phenomena we know as AUD. 
Table 1

Symptom Course as Defined by Endorsement Patterns

\begin{tabular}{cccc}
\hline & Prior to Wave 1 & Wave 1 & Wave 2 \\
Persistence & a & + & + \\
Onset & - & - & + \\
Recurrence & + & - & + \\
\hline
\end{tabular}

Note. "+" Represents the presence of the symptom at a given time point, "_-" represents the absence of a symptom at a given time.

${ }^{a}$ For persistence, lifetime endorsement prior to wave one was allowed but not required.

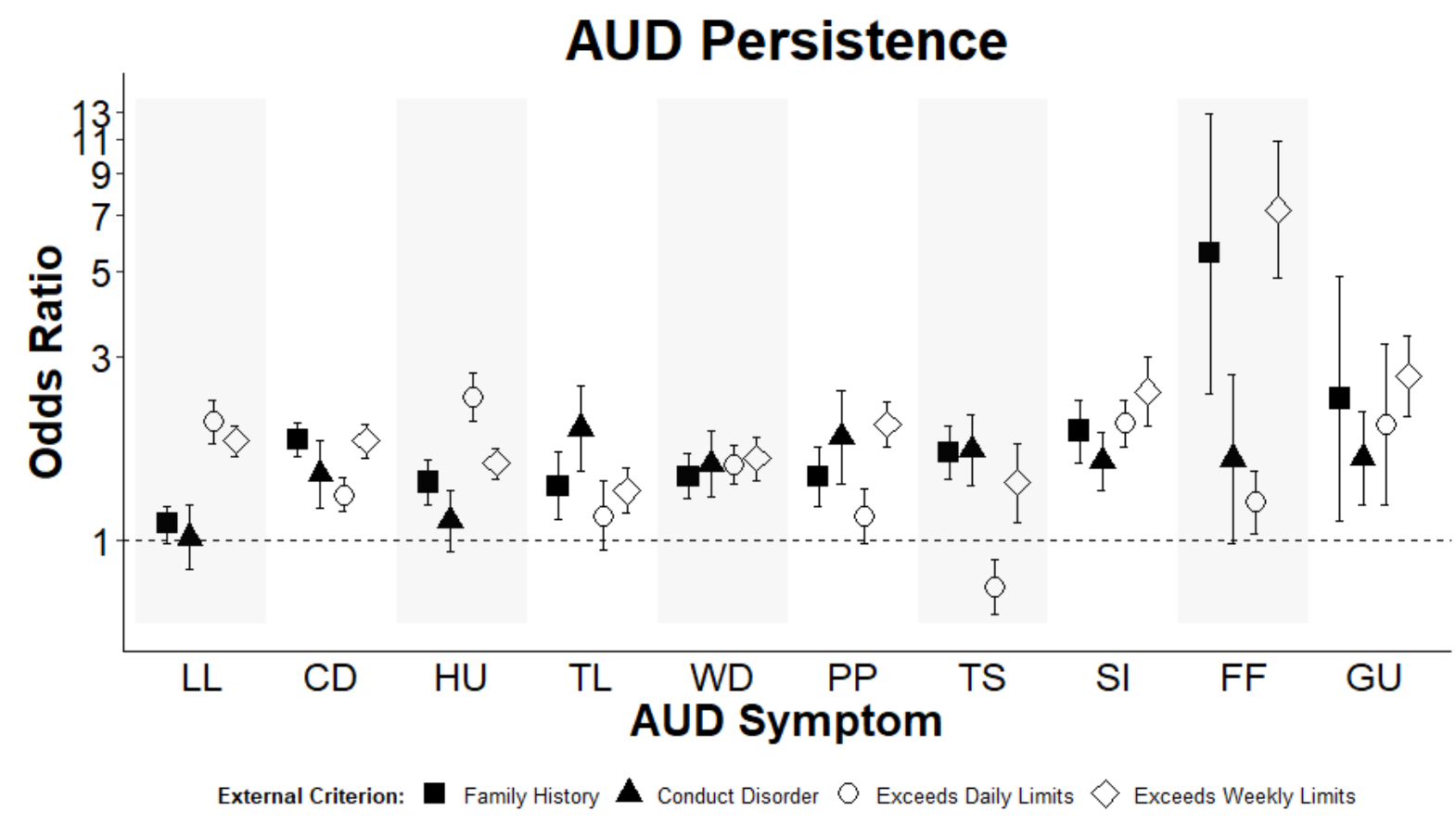

Error bars reflect $95 \%$ confidence intervals.

Figure 1. Persistence of AUD Symptoms. The Y axis is displayed using a logarithmic scale. 


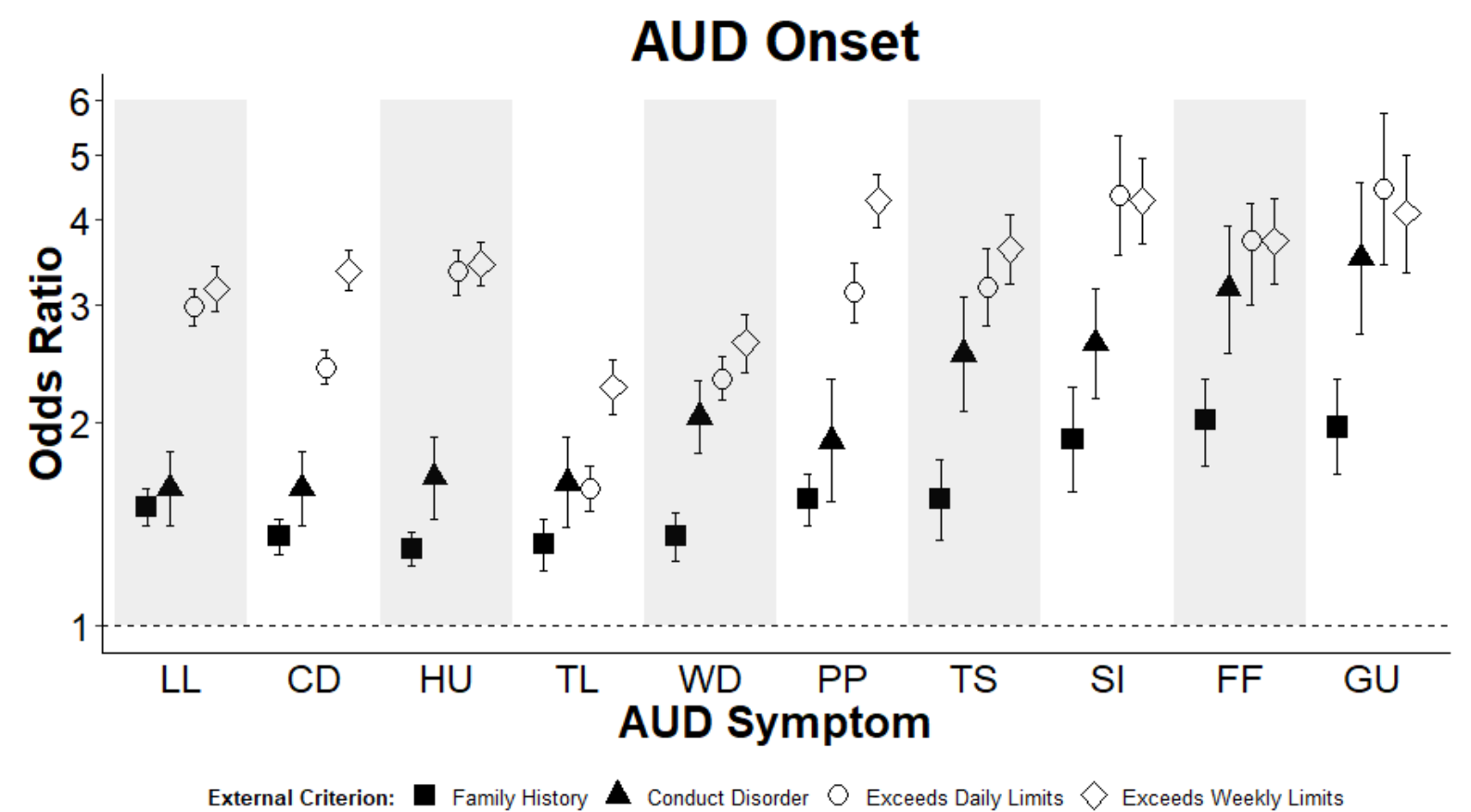

Error bars reflect $95 \%$ confidence intervals.

Figure 2. Onset of AUD Symptoms. The Y axis is displayed using a logarithmic scale.

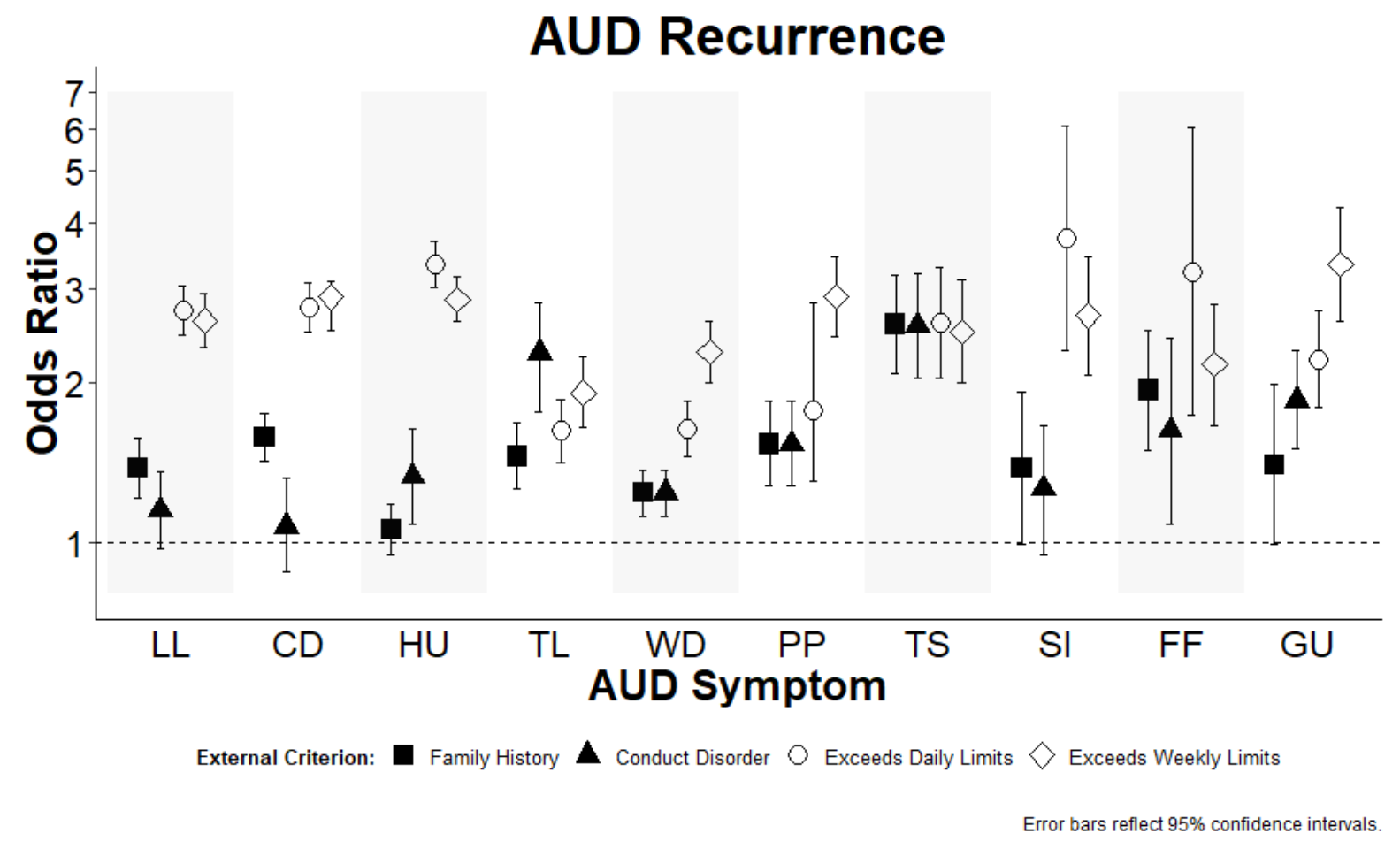

Figure 3. Recurrence of AUD Symptoms. The Y axis is displayed using a logarithmic scale. 


\section{References}

American Psychiatric Association (1994). Diagnostic and Statistical Manual of Mental Disorders, (4th ed.). Washington, DC: Author.

American Psychiatric Association. (2013). Diagnostic and statistical manual of mental disorders (DSM-5®). American Psychiatric Pub.

Andreasen, N. C., John, R., Jean, E., Theodore, R., \& William, C. (1986). The family history approach to diagnosis: how useful is it?.Archives of General Psychiatry, 43(5), 421-429. https://doi.org/10.1001/archpsyc.1986.01800050019002

Bickel, W. K., Crabbe, J. C., \& Sher, K. J. (2019). What is addiction? How can animal and human research be used to advance research, diagnosis, and treatment of alcohol and other substance use disorders? Alcoholism: Clinical and Experimental Research, 43(1), 6-21. https://doi.org/10.1111/acer.13912

Boness, C. L., Lane, S. P., \& Sher, K. J. (2016). Assessment of withdrawal and hangover is confounded in the Alcohol Use Disorder and Associated Disabilities Interview Schedule: Withdrawal prevalence is likely inflated. Alcoholism: Clinical and Experimental Research, 40(8), 1691-1699. https://doi.org/10.1111/acer.13121

Boness, C. L., Lane, S. P., \& Sher, K. J. (2019). Not all alcohol use disorder criteria are equally severe: Toward severity grading of individual criteria in college drinkers. Psychology of Addictive Behaviors, 33(1), 35-49. https://doi.org/10.1037/adb0000443 
Borsboom, D., \& Cramer, A. O. J. (2013). Network Analysis: An Integrative Approach to the Structure of Psychopathology. Annual Review of Clinical Psychology, 9(1), 91-121. https://doi.org/10.1146/annurev-clinpsy-050212-185608

Braun, P., Greenberg, D., Dasberg, H., \& Lerer, B. (1990). Core symptoms of posttraumatic stress disorder unimproved by alprazolam treatment. The Journal of Clinical Psychiatry, 51(6), 236238.

Bucholz, K. K., Heath, A. C., \& Madden, P. A. F. (2000). Transitions in drinking in adolescent females: evidence from the Missouri adolescent female twin study. Alcoholism: Clinical and Experimental Research, 24(6), 914-923. https://doi.org/10.1111/j.1530-0277.2000.tb02073.x

Bleuler, E. (1911). Dementia praecox oder Gruppe der Schizophrenien (Vol. 12). Deuticke.

Conradi, H. J., Ormel, J., \& De Jonge, P. (2011). Presence of individual (residual) symptoms during depressive episodes and periods of remission: a 3-year prospective study. Psychological Medicine, 41(6), 1165-1174. https://doi.org/10.1017/S0033291710001911

Chung, T., \& Martin, C. S. (2005). What were they thinking?: Adolescents' interpretations of DSMIV alcohol dependence symptom queries and implications for diagnostic validity. Drug and Alcohol Dependence, 80(2), 191-200. https://doi.org/10.1016/j.drugalcdep.2005.03.023

Davis, C. N., Slutske, W. S., Piasecki, T. M., Martin, N. G., \& Lynskey, M. T. (2020). Comparing the potential causal influence of two indicators of early alcohol use on later alcohol use disorder symptoms. Journal of Abnormal Psychology, 129(3), 256-265. https://doi.org/10.1037/abn0000474 
Grant, B. F., Dawson, D. A., \& Hasin, D. S. (2001). The alcohol use disorder and associated disabilities interview schedule-DSM-IV version. Bethesda, MD: National Institute on Alcohol Abuse and Alcoholism.

Grant, B. F., Kaplan, K. D., \& Stinson, F. S. (2005). Source and accuracy statement for the wave 2 National Epidemiologic Survey on Alcohol and Related Conditions (NESARC) Rockville. MD: National Institute on Alcohol Abuse and Alcoholism.

Grant, B. F., Moore, T. C., Shepard, J., \& Kaplan, K. (2003). Source and accuracy statement: Wave 1 national epidemiologic survey on alcohol and related conditions (NESARC). Bethesda, MD: National Institute on Alcohol Abuse and Alcoholism, 52.

Grant, B. F., Stinson, F. S., Dawson, D. A., Chou, S. P., Ruan, W. J., \& Pickering, R. P. (2004). Cooccurrence of 12-month alcohol and drug use disorders and personality disorders in the United States: results from the National Epidemiologic Survey on Alcohol and Related Conditions. Archives of General Psychiatry, 61(4), 361-368. https://doi.org/10.1001/archpsyc.61.4.361

Grant, J. D., Vergés, A., Jackson, K. M., Trull, T. J., Sher, K. J., \& Bucholz, K. K. (2012). Age and ethnic differences in the onset, persistence and recurrence of alcohol use disorder. Addiction, 107(4), 756-765. https://doi.org/10.1111/j.1360-0443.2011.03721.x

Green, B. L., Lindy, J. D., Grace, M. C., Gleser, G. C., Leonard, A. C., Korol, M., \& Winget, C. (1990). Buffalo Creek survivors in the second decade: Stability of stress symptoms. American Journal of Orthopsychiatry, 60(1), 43-54. https://doi.org/10.1037/h0079168 
Haeny, A. M., Littlefield, A. K., \& Sher, K. J. (2016). Limitations of lifetime alcohol use disorder assessments: A criterion-validation study. Addictive Behaviors, 59, 95-99. https://doi.org/10.1016/j.addbeh.2016.03.021

Hart, E. L., Lahey, B. B., Loeber, R., Applegate, B., \& Frick, P. J. (1995). Developmental change in attention-deficit hyperactivity disorder in boys: a four-year longitudinal study. Journal of Abnormal Child Psychology, 23(6), 729-749.

Hasin, D. S., O'Brien, C. P., Auriacombe, M., Borges, G., Bucholz, K., Budney, A., et al. (2013). DSM-5 criteria for substance use disorders: Recommendations and rationale. American Journal of Psychiatry, 170(8), 834-851. https://doi.org/10.1176/appi.ajp.2013.12060782

Heilig, M., \& Egli, M. (2006). Pharmacological treatment of alcohol dependence: target symptoms and target mechanisms. Pharmacology \& Therapeutics, 111(3), 855-876. https://doi.org/10.1016/j.pharmthera.2006.02.001

Hendershot, C. S., Wardell, J. D., Samokhvalov, A. V., \& Rehm, J. (2017). Effects of naltrexone on alcohol self-administration and craving: meta-analysis of human laboratory studies. Addiction Biology, 22(6), 1515-1527. https://doi.org/10.1111/adb.12425

Hoeppner, B. B., Kahler, C. W., \& Jackson, K. M. (2011). Evaluating the validity and utility of scaling alcohol consumption indices alongside AUD symptoms in treatment-seeking adolescents. Drug and Alcohol Dependence, 115(3), 196-204. https://doi.org/10.1016/j.drugalcdep.2010.10.023

Jellinek, E. M. (1960). The Disease Concept of Alcoholism. Hillhouse Press. 
Kazdin, A. E. (1983). Psychiatric diagnosis, dimensions of dysfunction, and child behavior therapy. Behavior Therapy, 14(1), 73-99. https://doi.org/10.1016/S0005-7894(83)80088-4

Koob, G. F., \& Le Moal, M. (2001). Drug addiction, dysregulation of reward, and allostasis. Neuropsychopharmacology, 24(2), 97-129. https://doi.org/10.1016/S0893-133X(00)00195-0

Koob, G. F. (2011). Neurobiology of addiction. Focus, 9(1), 55-65. https://doi.org/10.1176/foc.9.1.foc55

Kraepelin, E. (1907) Clinical Psychiatry; abstracted and adapted from 7th ed. of Psychiatrie (1904) by A. R. Diefendorf (Macmillan; reprinted, Thoemmes Press, 2002).

Lane, S. P., \& Sher, K. J. (2015). Limits of current approaches to diagnosis severity based on criterion counts: An example with DSM-5 alcohol use disorder. Clinical Psychological Science, 3(6), 819-835. https://doi.org/10.1177/2167702614553026

Lane, S. P., Steinley, D., \& Sher, K. J. (2016). Meta-analysis of DSM alcohol use disorder criteria severities: Structural consistency is only 'skin deep'. Psychological Medicine, 46(8), 1769-1784. https://doi.org/10.1017/S0033291716000404

Langenbucher, J., Martin, C. S., Labouvie, E., Sanjuan, P. M., Bavly, L., \& Pollock, N. K. (2000). Toward the DSM-V: The Withdrawal-Gate Model versus the DSM-IV in the diagnosis of alcohol abuse and dependence. Journal of Consulting and Clinical Psychology, 68(5), 799.

Larsson, J. O., Larsson, H., \& Lichtenstein, P. (2004). Genetic and environmental contributions to stability and change of ADHD symptoms between 8 and 13 years of age: a longitudinal twin study. Journal of the American Academy of Child \& Adolescent Psychiatry, 43(10), 1267-1275. https://doi.org/10.1037/0022-006X.68.5.799 
Lee, M. R., Boness, C. L., McDowell, Y. E., Vergés, A., Steinley, D. L., \& Sher, K. J. (2018). Desistance and severity of alcohol use disorder: A lifespan-developmental investigation. Clinical Psychological Science, 6(1), 90-105. https://doi.org/10.1177/2167702617736852

Leshner, A. I. (1997). Addiction is a brain disease, and it matters. Science, 278(5335), 45-47. https://doi.org/10.1126/science.278.5335.45

Levinson, C. A., Zerwas, S., Calebs, B., Forbush, K., Kordy, H., Watson, H., ... \& Runfola, C. D. (2017). The core symptoms of bulimia nervosa, anxiety, and depression: A network analysis. Journal of Abnormal Psychology, 126(3), 340-354. https://doi.org/10.1037/abn0000254

Lilienfeld, S. O. (2013). Is psychopathy a syndrome? Commentary on Marcus, Fulton, and Edens. Personality Disorders: Theory, Research, and Treatment, 4(1), 85-86. https://doi.org/10.1037/a0027544

Lüscher, C., Robbins, T. W., \& Everitt, B. J. (2020). The transition to compulsion in addiction. Nature Reviews Neuroscience, 1-17. https://doi.org/10.1038/s41583-020-0289-z

Martin, C. S., Langenbucher, J. W., Chung, T., \& Sher, K. J. (2014). Truth or consequences in the diagnosis of substance use disorders. Addiction, 109(11), 1773-1778. https://doi.org/10.1111/add.12615

McDowell, Y. E., Vergés, A., \& Sher, K. J. (2019). Are some AUD criteria more (or less) externalizing than others? Distinguishing alcohol use symptomatology from general externalizing psychopathology. Alcoholism: Clinical and Experimental Research, 43 (3), 483-496. https://doi.org/10.1111/acer.13952 
Moffitt, T. E., Caspi, A., Taylor, A., Kokaua, J., Milne, B. J., Polanczyk, G., \& Poulton, R. (2010). How common are common mental disorders? Evidence that lifetime prevalence rates are doubled by prospective versus retrospective ascertainment. Psychological Medicine, 40(6), 899-909. https://doi.org/10.1017/S0033291709991036

Morgenstern, M., DiFranza, J. R., Wellman, R. J., Sargent, J. D., \& Hanewinkel, R. (2016).

Relationship between early symptoms of alcohol craving and binge drinking 2.5 years later. Drug and Alcohol Dependence, 160, 183-189. https://doi.org/10.1016/j.drugalcdep.2016.01.008

National Institute on Alcohol Abuse and Alcoholism (2019). Retrieved from

https://www.niaaa.nih.gov/alcohol-health/overview-alcohol-consumption/alcohol-use-disorders

National Institute on Drug Abuse (2018). Retrieved from

https://www.drugabuse.gov/publications/drugs-brains-behavior-science-addiction/drug-misuseaddiction

Nivard, M. G., Dolan, C. V., Kendler, K. S., Kan, K. J., Willemsen, G., Van Beijsterveldt, C. E. M., ... \& Middeldorp, C. M. (2015). Stability in symptoms of anxiety and depression as a function of genotype and environment: a longitudinal twin study from ages 3 to 63 years. Psychological Medicine, 45(5), 1039-1049. doi:10.1017/S003329171400213X

Nutt, D., Wilson, S., \& Paterson, L. (2008). Sleep disorders as core symptoms of depression. Dialogues in Clinical Neuroscience, 10(3), 329-336.

O'Neill, S. E., \& Sher, K. J. (2000). Physiological alcohol dependence symptoms in early adulthood: A longitudinal perspective. Experimental and Clinical Psychopharmacology, 8(4), 493-508. https://doi.org/10.1037/1064-1297.8.4.493 
Piazza, P. V., \& Deroche-Gamonet, V. (2013). A multistep general theory of transition to addiction. Psychopharmacology, 229(3), https://doi.org/387-413. 10.1007/s00213-013-3224-4

Pfaltz, M. C., Michael, T., Grossman, P., Margraf, J., \& Wilhelm, F. H. (2010). Instability of physical anxiety symptoms in daily life of patients with panic disorder and patients with posttraumatic stress disorder. Journal of Anxiety Disorders, 24(7), 792-798. https://doi.org/10.1016/j.janxdis.2010.06.001

Rohde, P., Lewinsohn, P. M., Kahler, C. W., Seeley, J. R., \& Brown, R. A. (2001). Natural course of alcohol use disorders from adolescence to young adulthood. Journal of the American Academy of Child \& Adolescent Psychiatry, 40(1), 83-90. https://doi.org/10.1097/00004583-20010100000020

Robinson, T. E., \& Berridge, K. C. (1993). The neural basis of drug craving: an incentivesensitization theory of addiction. Brain Research Reviews, 18(3), 247-291. https://doi.org/10.1016/0165-0173(93)90013-P

Saha, T. D., Stinson, F. S., \& Grant, B. F. (2007). The role of alcohol consumption in future classifications of alcohol use disorders. Drug and Alcohol Dependence, 89(1), 82-92. https://doi.org/10.1016/j.drugalcdep.2006.12.003

Schuckit, M. A., \& Smith, T. L. (2011). Onset and course of alcoholism over 25 years in middle class men. Drug and Alcohol Dependence, 113(1), 21-28. https://doi.org/10.1016/j.drugalcdep.2010.06.017

Schuckit, M. A., Clarke, D. F., Smith, T. L., Mendoza, L. A., \& Schoen, L. (2020). The search for contributors to low rates of recognition of paternal alcohol use disorders in offspring from the 
San Diego Prospective Study. Alcoholism: Clinical and Experimental

Research. 44(8), 1551-1560. https://doi.org/10.1111/acer.14401

Timko, C., Finney, J. W., \& Moos, R. H. (2005). The 8-year course of alcohol abuse: gender differences in social context and coping. Alcoholism: Clinical and Experimental Research, 29(4), 612-621. https://doi.org/10.1097/01.ALC.0000158832.07705.22

Tuithof, M., ten Have, M., van den Brink, W., Vollebergh, W., \& de Graaf, R. (2014). Alcohol consumption and symptoms as predictors for relapse of DSM-5 alcohol use disorder. Drug and Alcohol Dependence, 140, 85-91. https://doi.org/10.1016/j.drugalcdep.2014.03.035

Vandiver, T., \& Sher, K. J. (1991). Temporal stability of the Diagnostic Interview Schedule. Psychological Assessment: A Journal of Consulting and Clinical Psychology, 3(2), 277-281. https://doi.org/10.1037/1040-3590.3.2.277

Van Borkulo, C., Boschloo, L., Borsboom, D., Penninx, B. W., Waldorp, L. J., \& Schoevers, R. A. (2015). Association of symptom network structure with the course of depression. JAMA Psychiatry, 72(12), 1219-1226. https://doi.org/10.1001/jamapsychiatry.2015.2079

Vergés, A., Lee, M. R., Martin, C. S., Trull. T. J., Martens, M. P., Wood, P. K., \& Sher, K. J. (under review). Not all symptoms of alcohol dependence are developmentally equivalent: Implications for the false positives problem.

Volpicelli, J. R., Alterman, A. I., Hayashida, M., \& O'Brien, C. P. (1992). Naltrexone in the treatment of alcohol dependence. Archives of General Psychiatry, 49(11), 876-880. https://doi.org/10.1001/archpsyc.1992.01820110040006 


\section{Footnotes}

${ }^{1}$ Tolerance $=$ "Tolerance, as defined by either of the following: (a) A need for markedly increased amounts of alcohol to achieve intoxication or desired effect (b) A markedly diminished effect with continued use of the same amount of alcohol." Quit/Cut Down = "There is a persistent desire or unsuccessful efforts to cut down or control alcohol use;" Larger/Longer = "Alcohol is taken in larger amounts or over longer periods than was intended;" Time Spent = "A great deal of time is spent in activities necessary to obtain alcohol, use alcohol, or recover from its effects;" Craving = "Craving, or a strong desire or urge to use alcohol;" Give Up = "Important social, occupational, or recreational activities given up or reduced because of alcohol use;” Physical/Psychological $=$ "Alcohol use is continued despite knowledge of having a persistent or recurrent physical or psychological problem that is likely to have been caused or exacerbated by alcohol." Withdrawal = "Withdrawal, as manifested by either of the following: (a) The characteristic withdrawal syndrome for alcohol, (b) Alcohol (or a closely related substance, such as benzodiazepine) is taken to relieve or avoid withdrawal symptoms." Hazardous Use = "Recurrent alcohol use in situations in which it is physically hazardous." Social/Interpersonal = "Continued alcohol use despite having persistent or recurrent social or interpersonal problems caused or exacerbated by the effects of alcohol." Failure to Fulfill = "Recurrent alcohol use resulting in a failure to fulfill major role obligations at work, school, or home;" 
Correspondence concerning this article should be addressed to William Conlin, Department of Psychological Science, Psychology Building, 200 South Seventh Street, Columbia, MO 65211. Phone: +1928978 7217. E-mail: wecxk6@umsystem.edu 